\title{
A EXPRESSÃO DA GENERICIDADE NO PORTUGUÊS DO BRASIL*
}

Ana Müller**

\section{Introdução}

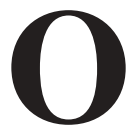

objetivo deste trabalho é investigar a semântica das expressões de referência a espécie e das sentenças genéricas no Português do Brasil ( $\mathrm{PB})$. Mais especificamente, vou investigar as diferenças entre as interpretações do definido genérico singular e plural, do indefinido genérico, do singular nu e do plural nu. Somente os nomes contáveis serão investigados. A análise servirá de ponto de partida para uma comparação com o comportamento desse fenômeno no Português Europeu (PE).

A hipótese central é a de que a denotação do nome comum no PB inclui tanto átomos como pluralidades. As diferenças na interpretação dos vários sintagmas genéricos e sentenças genericamente quantificadas resultam desse

* Diferentes versões desse trabalho foram apresentadas no Proseminar in Semantics 1999, UMass-Amherst, USA, nos Seminários em Teoria Gramatical, USP-SP, no Colóquio PE-PB 2000, Coimbra, Portugal e no GT de Semântica do Celsul 2000, Curitiba, PR. Agradeço os comentários dos participantes, em particular, agradeço os comentários detalhados de Barbara Partee.

A primeira versão desse trabalho foi elaborada durante meu pós-doutorado junto à UMassAmherst, EUA apoiado pela Capes e pela Fapesp. Agradeço a essas instituições.

** Universidade de São Paulo 
fato e da existência de um operador singular (SING) e de um operador plural (PL) sobre a denotação do nome comum.

$\mathrm{O}$ artigo segue a seguinte seqüência:

(i) Na seção 1, apresento os fatos descritivos sobre a expressão da genericidade no PB assumindo que as línguas naturais fazem uso de dois mecanismos diferentes para expressar a genericidade: expressões de referência a espécie e quantificação genérica.

(ii) $\mathrm{Na}$ seção 2, proponho uma interpretação para os nomes comuns e a interpretação para um operador singular (SING) e um operador plural (PL) que postulo existir nessa língua.

(iii) Na seção 3, aplico a proposta desenvolvida para derivar as formas lógicas de sentenças com genéricos indefinidos.

(iv) $\mathrm{Na}$ seção 4, discuto a interpretação dos definidos genéricos.

\section{Expressões de referência a espécies vs. sentenças genericamente quantificadas}

Nesta seção, vou considerar em detalhe o comportamento de argumentos genéricos em PB. O ponto de partida teórico é o fato de que as línguas naturais fazem uso de dois mecanismos distintos para expressar genericidade: expressões de referência a espécie - expressões que denotam diretamente uma espécie, e quantificação genérica sobre sentenças - sentenças sob o escopo de um operador de genericidade.

Vários tipos de expressões genéricas podem ser usadas em PB, como se pode ver nas sentenças (1) a (5).

(1) O automóvel chegou ao Brasil no século XX.

(2) As cobras são animais perigosos.

(3) Um número par é sempre divisível por dois.

(4) Homem não chora.

(5) Professores trabalham muito. 
Vou agora fazer uso de alguns testes propostos por Krifka et al. (1995) e Gestner e Krifka 1993 para tentar distingüir entre os dois tipos de genericidade no PB.

(i) Existem alguns predicados com posições argumentais que podem ser preenchidas apenas por expressões de referência a espécies como estar extinto.

(6) O mico-leão-dourado está extinto.

(7) Os micos-leões-dourados estão extintos.

(8) *Um mico-leão-dourado está extinto.

(9) *Mico-leão-dourado está extinto.

(10) *Micos-leões-dourados estão extintos.

(11) Um (tipo de) mico-leão-dourado está extinto.

(ii) Sentenças genéricas são estativas. Conseqüentemente, uma sentença com um predicado episódico só poderá expressar algum tipo de genericidade se um de seus argumentos for uma expressão de referência a espécie. ${ }^{1}$

(12) O automóvel chegou ao Brasil no século XX.

(13) Os automóveis chegaram ao Brasil no século XX.

(14) *Um automóvel chegou ao Brasil no século XX.

(15) *Automóvel chegou ao Brasil no século XX.

(16) *Automóveis chegaram ao Brasil no século XX.

(iii) Expressões de referência a espécie parecem estranhas quando construídas a partir de espécies não-estabelecidas.

(17) a. A garrafa de coca tem gargalo estreito.

b. ???A garrafa verde tem gargalo estreito.

(18) a. As garrafas de coca têm gargalo estreito.

b. ??As garrafas verdes têm gargalo estreito.

(19) a. Uma garrafa de coca tem gargalo estreito.

b. Uma garrafa verde tem gargalo estreito.

1 As sentenças (12) a (16) têm que ser lidas como se referindo a um único evento de chegada. 
(20) a. Garrafa de coca tem gargalo estreito.

b. Garrafa verde tem gargalo estreito.

(21) a. Garrafas de coca têm gargalo estreito.

b. Garrafas verdes têm gargalo estreito.

A partir do comportamento das expressões genéricas do PB em relação a (i) a (iii) podemos estabelecer duas generalizações atraentes sobre a expressão da genericidade em PB:

- O definido genérico singular e o definido genérico plural são expressões de referência a espécies.

- O indefinido genérico, o singular nu e o plural nu não denotam espécies. Sua genericidade deve provir de sua participação em sentenças genericamente quantificadas.

\section{A interpretação dos nomes comuns e dos morfemas singular e plural}

O objetivo desta seção é explicitar uma proposta para a interpretação dos nomes comuns em PB. Minha hipótese é que a denotação de um nome comum no PB é uma neutralização entre o singular e o plural. Essa hipótese é semelhante à denotação proposta por Chierchia 1998 para nomes massivos e à denotação que Link 1983 atribui aos predicados plurais. Um nome comum teria como parte de sua denotação todas as entidades atômicas e todas as entidades plurais (todas as somas de qualquer número de entidades).

Por exemplo, em um universo com apenas três bolachas, a denotação de bolacha seria como em (1).

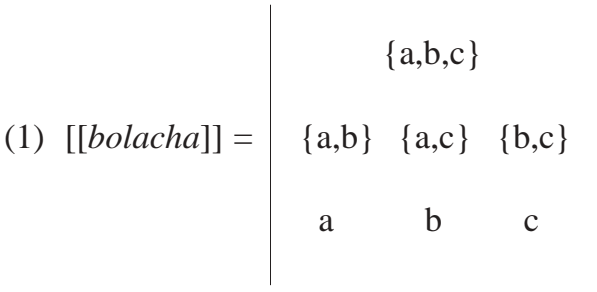


Como o PB possui morfologia de número (2 e 3 ) e essa morfologia tem significado semântico, os morfemas de número podem ser analisados como operadores sobre o nome comum (ou sobre o NP). ${ }^{2}$ Estes operadores estão apresentados em (4) e (5) e seu funcionamento é exemplificado em (6) e (7).

(2) a bolacha(sg)/aS bolachaS(pl) (dialeto culto)

(3) a bolacha/aS bolacha (dialeto popular)

(4) $\operatorname{SING}(\mathrm{ular})=\lambda \mathrm{P} \lambda \mathrm{x}[\mathrm{P}(\mathrm{x}) \wedge A t(\mathrm{x})]^{3}$

(5) $P L($ ural $)=\lambda \mathrm{P} \lambda \mathrm{x}[\mathrm{P}(\mathrm{x}) \wedge \neg A t(\mathrm{x})]$

(6) $\operatorname{SING}($ bolacha $)=\lambda \mathrm{P} \lambda \mathrm{x}[\mathrm{P}(\mathrm{x}) \wedge A t(\mathrm{x})]($ bolacha $)$

$=\lambda \mathrm{x}[$ bolacha $(\mathrm{x}) \wedge A t(\mathrm{x})]$

(7) $\mathrm{PL}($ bolacha $)=\lambda \mathrm{P} \lambda \mathrm{x}[\mathrm{P}(\mathrm{x}) \wedge \neg A t(\mathrm{x})]($ bolacha $)$

$=\lambda \mathrm{x}[$ bolacha $(\mathrm{x}) \wedge \neg A t(\mathrm{x})]$

Como ilustram (8) e (9), o operador plural (PL) retira todos os átomos da denotação de um nome comum e o operador singular (SING) retira-lhe todas as entidades não-atômicas.

(8) $[$ (bolacha) $]=$

(9) $[\operatorname{sing}($ bolacha $)]=$

$|\mathrm{a} \quad| \begin{array}{lll} & \mathrm{a} \oplus \mathrm{b} \oplus \mathrm{c} & \\ & \mathrm{a} \oplus \mathrm{c} & \\ & & \\ \mathrm{a} \oplus \mathrm{b} & & \mathrm{b} \oplus \mathrm{c}\end{array} \mid$

Dados empíricos que confirmam essa análise:

- As três formas de indefinido são diferentes quanto a seu número semântico. Isso aparece quando o indefinido está em posição de objeto. (Portanto, o PB não parece ter plurais dependentes.)

(10) Unicórnio tem um chifre (apenas um chifre).

(11) Unicórnio tem chifre (um número não especificado de chifres).

(12) Unicórnio tem chifres (mais de um chifre).

2 Estou assumindo a seguinte estrutura para o sintagma de determinante: [DP ...[NP ...[N...]]].

$3[[$ A $t$ a $]]:=1$ se [[a]] é atômico. 
- Todos os tipos de sujeitos indefinidos podem ser usados com todos os tipos de objetos indefinidos com o mesmo efeito sobre o número. ${ }^{4}$ Portanto, esse não parece ser um fenômeno sintático.

(13) Um unicórnio tem um chifre/chifre/chifres.

(14) Unicórnios têm um chifre/chifre/chifres.

- O comportamento com recíprocos e anáforas confirma a hipótese, pois não há átomos definidos a serem retomados em (15) e seu retoma uma pluralidade em (16).

(15) *Brasileiro detesta um ao outro.

(16) Os cães perderam seus rabos (mais de um rabo!!).

Outros argumentos para que o singular simples seja considerado como não especificado para número vem de Schmitt and Munn 1999. Sabe-se que as propriedades do objeto direto afetam o aspecto do sintagma verbal de maneira que objetos quantizados causam leituras terminativas, enquanto objetos não quantizados causam leituras durativas.

- Singulares simples permitem apenas leituras durativas.

(17) Eu escrevi carta por duas horas.

(18) *Eu escrevi carta em duas horas. ${ }^{5}$

\section{Indefinidos participando em sentenças genericamente quantificadas: uma análise do indefinido genérico, do singular nu e do plural nu}

Nesta seção vou explorar as maneiras pelas quais o PB expressa quantificação genérica em relação à proposta de que nomes comuns devem ser considerados não especificados para número nessa língua.

Indefinidos genéricos não são verdadeiras expressões genéricas porque não denotam espécies. Primeiro, eles ocorrem apenas em sentenças genericamente quantificadas. Segundo, eles não podem ser usados com predicados que

4 Leituras coletivas são possíveis, mas não serão consideradas neste trabalho.

5 Essa sentença se torna aceitável se receber algum tipo de leitura iterativa, como: Eu estava tão ocupada naquela época que eu preparei aula em uma hora. 
aplicam-se apenas a espécies. Finalmente, eles não podem ser usados com predicados que não são estativos. Veja os exemplos (1) a (6) do inglês:

(1) The potato was first cultivated in South America. ${ }^{6}$

(2) *A potato was first cultivated in South America. ${ }^{7}$

(3) Graham Bell invented the telephone.

(4) *Graham Bell invented a telephone.

(5) The rat was reaching Australia in 1970.

(6) *A rat was reaching Australia in 1970.

Muitos dos trabalhos recentes sobre a genericidade adotam uma análise de sentenças genericamente quantificadas como sentenças contendo um operador relacional genérico (GEN) que toma duas sentenças, uma restrição e uma matriz, como seus argumentos, como em (7), que está ilustrado em (8). ${ }^{8}$

Versão simplificada:

(7) GEN [x; y] (Restrição [x]; Matriz [x, y])

$=$ GEN $[\mathrm{x}]($ Restrição $[\mathrm{x}] ; \exists \mathrm{y}$ Matriz $[\mathrm{x}, \mathrm{y}])$

('Genericamente, se restrição $\mathrm{x}$, então existe um y tal que matriz x,y')

Exemplo:

(8) a. Brasileiro dança bem

b. GEN [x; ] (x é Brasileiro; x dança bem)

c. 'Tipicamente, se alguém é brasileiro, então dança bem'

Vou tratar Sintagmas de Determinantes (DPs) indefinidos como indefinidos heimianos, ou seja, como fórmulas contendo uma variável livre (um brasileiro $=$ brasileiro $(\mathrm{x})$ ) (cf. Heim 1982). Se o indefinido singular, o singular nu e o plural nu são todos indefinidos 'normais' no PB, eles adquirem seu significado genérico do fato de estarem participando de sentenças genericamente quantificadas nas quais suas variáveis são ligadas pelo operador GEN.

Vou agora tratar a questão de quais as diferenças entre as interpretações dos vários tipos de indefinidos em Рв. Vou exemplificar, por simplicidade, com uma sentença habitual (9).

6 As sentenças (1)-(6) são de Krifka et al., 1995.

7 O asterisco, como anteriormente, indica má-formação semântica e não sintática. Na verdade, ele marca apenas que essas sentenças não podem ser interpretadas como equivalentes genéricos de (1), (3) e (5). Elas poderiam ser compreendidas como genéricas sob a leitura 'shifted' 'um tipo de...'

8 Veja os trabalhos em Carlson e Pelletier 1995. 
(9) Depois do jantar, Jorge come uma bolacha/bolacha/bolachas.

Em (10) temos a interpretação de uma sentença com o singular nu:

(10) a. Depois do jantar, Jorge come bolacha. (um número indefinido de bolachas)

b. GEN $[\mathrm{s} ; \mathrm{x}]$ (Jorge em $\mathrm{s} \wedge$ depois do jantar $\mathrm{s}$; bolacha $\mathrm{x} \wedge$ Jorge come $\mathrm{x}$ em $\mathrm{s})$

$=G E N[\mathrm{~s} ;]($ Jorge em $\mathrm{s} \wedge$ depois do jantar $\mathrm{s} ; \exists \mathrm{x}$ (bolacha $\mathrm{x}$ Jorge come $\mathrm{x}$ em $\mathrm{s}))^{9}$

Em (11) e (12) temos o cálculo da interpretação de uma sentença com o indefinido genérico:

(11) uma bolacha $=$ uma $(\operatorname{sing}($ bolacha $))=\lambda \mathrm{x}[$ bolacha $(\mathrm{x}) \wedge A t(\mathrm{x})]$

(12) a. Depois do jantar, Jorge come uma bolacha. (apenas uma bolacha)

b. GEN $[\mathrm{s} ; \mathrm{x}]$ (Jorge em $\mathrm{s} \wedge$ depois do jantar s; uma (SING (bolacha)) $\mathrm{x} \wedge$ Jorge come $\mathrm{x}$ em $\mathrm{s}$ )

$=G E N[\mathrm{~s} ; \mathrm{x}]($ Jorge em $\mathrm{s}$ Ù depois do jantar $\mathrm{s} ; \lambda \mathrm{y}[$ bolacha $(\mathrm{y}) \wedge$ At (y)] (x) $\wedge$ Jorge come $\mathrm{x}$ em $\mathrm{s})$

$=$ GEN $[\mathrm{s} ;]$ (Jorge em $\mathrm{s} \wedge$ depois do jantar $\mathrm{s} ; \exists \mathrm{x}(\lambda \mathrm{y}[$ bolacha $(\mathrm{y}) \wedge$ At $(\mathrm{y})](\mathrm{x}) \wedge \wedge$ Jorge come $\mathrm{x}$ em $\mathrm{s}))$

$=G E N[\mathrm{~s} ;]($ Jorge em $\mathrm{s} \wedge$ depois do jantar $\mathrm{s} ; \exists \mathrm{x}($ bolacha $(\mathrm{x}) \wedge A t(\mathrm{x})$ $\wedge$ Jorge come $\mathrm{x} \mathrm{em} \mathrm{s))}$

Para obtermos a interpretação do plural nu, precisamos aplicar o operador plural (PL) a(a denotação d)o nome comum, como se pode ver em (13).

(13) a. Depois do jantar, Jorge come bolachas. (mais de uma bolacha).

b. GEN $[\mathrm{s} ; \mathrm{x}]$ (Jorge em $\mathrm{s} \wedge$ depois do jantar $\mathrm{s}$; bolachas $(\mathrm{x}) \wedge$ Jorge come $\mathrm{x}$ em $\mathrm{s}$ $=G E N[\mathrm{~s} ; \mathrm{x}]($ Jorge em $\mathrm{s} \wedge$ depois do jantar $\mathrm{s} ;$ PL $($ bolacha $)(\mathrm{x}) \wedge$ Jorge come $\mathrm{x}$ em $\mathrm{s}$ $=G E N[\mathrm{~s} ;]($ Jorge em $\mathrm{s} \wedge$ depois do jantar $\mathrm{s} ; \exists \mathrm{x}($ ly [bolacha $(\mathrm{y}) \wedge$ $\neg A t(\mathrm{y})](\mathrm{x}) \wedge$ Jorge come $\mathrm{x}$ em $\mathrm{s}))$ $=G E N[\mathrm{~s} ;]($ Jorge em $\mathrm{s} \wedge$ depois do jantar $\mathrm{s} ; \exists \mathrm{x}($ bolacha $(\mathrm{x}) \wedge \neg A t$ (x) $\wedge$ Jorge come $\mathrm{x} \mathrm{em} \mathrm{s))}$

9 s é uma variável sobre situações. Já o quantificador existencial está sempre presente e liga quaisquer variáveis ainda livres na matriz (ver Krifka et al. 1995 e Heim 1982). 


\section{Expressões de referência à espécie no português brasileiro}

Em PB tanto o definido singular quanto o definido plural comportam-se como expressões de referência a espécies. Esta seção discute a questão de como a denotação proposta para o nome comum, os operadores plural (PL) e singular (SING) e o artigo definido se compõe para resultar em uma interpretacão de espécie no PB.

As sentenças (1) e (2) ilustram os dois modos de se expressar genericidade: sentenças genericamente quantificadas e expressões de referência a espécies. Apesar de ambas as sentenças possuirem significados extremamente próximos, as suas interpretações resultam de dois processos distintos.

(1) a. Brasileiro dança bem.

"Geralmente, se alguém é brasileiro, dança bem"

b. GEN [brasileiro x; dança bem x]

(2) a. $O$ brasileiro dança bem.

"A espécie BRASILEIRO tem a propriedade de dançar bem" b. dança-bem (BRASILEIRO)

Como já vimos, a expressão da genericidade no caso de expressões de referência a espécies é uma propriedade do Sintagma de Determinante, não da sentença. SDs que são expressões de referências a espécie são capazes de coocorrer com qualquer tipo de predicado:

(3) O mico-leão-dourado logo estará extinto. (predicado de espécie)

(4) O mico-leão-dourado é inteligente. (predicado de indivíduo)

(5) $O$ brasileiro está fumando muito. (predicado de estágio)

\section{Um artigo definido genérico}

A idéia central desta seção é a de que o artigo definido genérico seleciona a única espécie (ao invés do único indivíduo) à qual pertencem todas as realizações de um predicado. Espécies são (um certo tipo de) indivíduos. Expressões de referência a espécie são expressões que se referem a essas entidades. Temos então que MICO-LEÃO-DOURADO denota a espécie correspondente ao predicado mico-leão-dourado como em (1). 
(1) a. O mico-leão-dourado está extinto.

b. está-extinto (MICO-LEÃO-DOURADO)

Há uma relação entre uma espécie e seu predicado - a relação $R$ que é a relação de realização (2) (cf. Carlson 1977).

(2) $R(\mathrm{y}, \mathrm{x}):$ o objeto y pertence à espécie $\mathrm{x}$.

O artigo definido genérico em (3) seleciona então a única espécie à qual pertencem todas as entidades denotadas por um nome comum ou um sintagma nominal (NP), como ilustrado em (4). A denotação de uma sentença com um DP definido genérico está detalhada em (5).

(3) $\mathrm{o}_{\text {GENÉRICO }}=\lambda \mathrm{Q}$ ix $\quad \forall \mathrm{y}[\mathrm{Q}(\mathrm{y}) \leftrightarrow R(\mathrm{y}, \mathrm{x})]$

(4) o mico-leão-dourado $=\mathrm{o}_{\text {GENÉRICO }}$ (mico-leão-dourado)

$=\lambda \mathrm{Q} i \mathrm{x} \forall \mathrm{y}[\mathrm{Q}(\mathrm{y}) \leftrightarrow R(\mathrm{y}, \mathrm{x})]$ (mico-leão-dourado)

$=1 \mathrm{x} \forall \mathrm{y}[$ mico-leão-dourado $(\mathrm{y}) \leftrightarrow R(\mathrm{y}, \mathrm{x})]$

(5) está-extinto $\{\mathrm{ix} \forall \mathrm{y}[$ mico-leão-dourado $(\mathrm{y}) \leftrightarrow R(\mathrm{y}, \mathrm{x})]\}$

Quando se aplica essa idéia ao PB, deve-se levar em conta a denotação proposta para os nomes comuns e a existência dos operadores singular (SING) e plural (PL). O funcionamento desses operadores em conjunto com o artigo definido genérico está ilustrado na sentença (6).

(6) a. Os micos-leões-dourados estão extintos.

b. estão-extintos $\left\{\mathrm{O}_{\mathrm{GENÉ}}[P L\right.$ (mico-leão-dourado $\left.\left.)\right]\right\}$

$=$ estão-extintos $\{\mathrm{lx} \forall \mathrm{y}[($ mico-leão-dourado $(\mathrm{y}) \wedge \neg A t(\mathrm{y})) \leftrightarrow$ $R(\mathrm{y}, \mathrm{x})]\}$

Algumas consequiências da proposta são bem-vindas:

- A sentença (6) seria verdadeira ainda que houvesse apenas um micoleão-dourado no mundo.

- A diferença entre as denotações do singular e do plural genérico po- 
dem nos ajudar a explicar porque (7a) não é uma boa sentença para uma interpretação genérica não-taxonômica. Sua forma lógica (7b) afirma que a espécie que Graham Bell inventou nunca é realizada como uma entidade atômica e isso não corresponde ao nosso conhecimento de mundo.

(7) a. Graham Bell inventou os telefones.

b. inventou (GB, $\in \mathrm{x} \forall \mathrm{y}[($ telefone $(\mathrm{y})$ Ù $\emptyset A t(\mathrm{y})) \ll R(\mathrm{y}, \mathrm{x})]$ )

Resumindo:

- O comportamento da genericidade no PB confirma a visão de que sua expressão faz uso de (pelo menos) dois mecanismos diferentes: sentenças genericamente quantificadas e expressões de referência a espécie.

- Genéricos indefinidos no PB - indefinidos genéricos, singulares nus e plurais nus - são apenas indefinidos heimianos 'normais' participando de sentenças genericamente quantificadas. Sua genericidade provem do fato de que eles têm suas variáveis presas pelo operador genérico.

- Definidos Genéricos são expressões de referência a espécies em PB. Elas são formadas pela aplicação do artigo definido genérico a um nome comum que foi "sINGularizado" ou "PLuralizado".

- Nomes comuns não são especificados para número em PB. Sua denotação inclui tanto indivíduos singulares quanto plurais. 


\section{RESUMO}

A genericidade no PB faz uso de dois mecanismos: as sentenças genericamente quantificadas e as expressões de referência à espécie. Os genéricos indefinidos são indefinidos no sentido de Heim, isto é, são variáveis presas pelo operador genérico. Os definidos genéricos referem-se a espécies e resultam do artigo definido genérico aplicado a um nome comum singularizado ou pluralizado. Nomes comuns são denotacionalmente neutros.

Palavras-chaves: Genericidade, nomes comuns, semântica.

\section{ABSTRACT}

Genericity in Brazilian Portuguese is expressed by two mechanisms: generically quantified sentences and species referring expressions. Indefinites generics are indefinite in Heim's sense, they are variables bound by the generic operator. Generic definites refer to species and they result from applying the generic definite article to a common noun, which is taken in the singular or in the plural. Common nouns are denotationally neutral.

Key-words: Genericity, common nouns, semantics.

\section{REFERÊNCIAS}

CARLSON, G.; PELLETIER, F. J. (Ed.). The generic book. Chicago \& London: The University of Chicago Press, 1995.

CHIERCHIA, G. Reference to kinds across languages. Natural language semantics, n. 6, p. 339-405, 1998.

GESTNER, C.; KRIFKA, N. Genericity. In: JACOBS, J.et al. (Eds.). Handbuch der Syntax, Berlim: de Gruyter, p. 966-978, 1993. 
HEIM, I. The Semantics of definite and indefinite noun phrases. UMass, $\mathrm{PhD}$. dissertation, 1982.

KRIFKA, M. et al. Genericity: an Introduction. In: CARLSON, G.; PELLETIER, F. J. (Eds.). The generic book. Chicago \& London: The University Chicago Press, 1995. p. 1124.

LINK, G. The logical analysis of plurals and mass terms: a lattice theoretical approach. In: BÄUERLE, R.; SCHWARZE, C.; STECHOW, A. Von. (Eds.). Meaning, use and interpretation of language. Berlin: de Gruyter, 1983. p. 303-323. 
